\title{
Dual wavelength digital holography for 3D particle image velocimetry
}

S. Grare

grares@coria.fr

\section{S. Coëtmellec \\ D. Allano}

G. Grehan

\section{Brunel \\ D. Lebrun}

\author{
Département optique-lasers, UMR-6614 CORIA, LABEX EMC3, Av. de l'Université, 76801 Saint- \\ Etienne du Rouvray cedex, France \\ Département optique-lasers, UMR-6614 CORIA, LABEX EMC3, Av. de l'Université, 76801 Saint- \\ Etienne du Rouvray cedex, France \\ Département optique-lasers, UMR-6614 CORIA, LABEX EMC3, Av. de l'Université, 76801 Saint- \\ Etienne du Rouvray cedex, France \\ Département optique-lasers, UMR-6614 CORIA, LABEX EMC3, Av. de l'Université, 76801 Saint- \\ Etienne du Rouvray cedex, France \\ Département optique-lasers, UMR-6614 CORIA, LABEX EMC3, Av. de l'Université, 76801 Saint- \\ Etienne du Rouvray cedex, France \\ Département optique-lasers, UMR-6614 CORIA, LABEX EMC3, Av. de l’Université, 76801 Saint- \\ Etienne du Rouvray cedex, France
}

A multi-exposure digital in-line hologram of a moving particle field is recorded by two different wavelengths and at different times. As a result, during the reconstruction step, each hologram can be independently and accurately reconstructed for each wavelength. This procedure enables avoiding the superimposition of particles images that may be close to each other in multi-exposure holography. The feasibility is demonstrated by using a standard particle sizing reticle and shows the potential of this method for particle velocity measurement.

[DOI: http://dx.doi.org/10.2971/jeos.2015.15009]

Keywords: Holography, image reconstruction techniques, three dimensional particle image velocimetry

\section{INTRODUCTION}

The use of digital holography for particle velocity measurement is recognized nowadays [1]-[5]. A significant number of articles deal with the different optical configurations or different methods for hologram reconstruction [6]-[9]. When both the velocity and the trajectories of the particles are wanted, it is also possible to record holograms using multiple exposures [10]-[12]. In this situation, knowing the mean velocity of the flow, the time interval between pulses must be large enough to correctly separate the reconstructed particle images [13]. However, in real experimental situations, the flows to be studied exhibit a wide range of velocities. Thus, it is difficult to find a time interval well suited for the whole displacements between pulses. In particular, the velocities of the slowest particles cannot be safely measured due to the impossibility of separating an image pair. Moreover, the sign of the velocity vector is unknown. A possible way to remove the ambiguity in the direction consists in recording holograms separately on two different frames as in Particle Image Velocimetry [14]. Here we propose to record holograms in the same frame but with two different wavelengths. In other words, the role played by the camera in standard PIV to split each image into two separate frames is replaced here by a wavelength coding. During the reconstruction step, a couple of images are obtained for each colour (or a couple of images spatially separated in depth if a single reconstruc- tion wavelength is used). The recording and reconstruction of two-colour in-line holograms is given in Section 2. In particular, the method used for focusing on each image is presented. The results of an experiment to demonstrate the feasibility by using a standard reticle of particles are presented in Section 3 before the presentation of some brief conclusions in Section 4.

\section{HOLOGRAM RECORDING WITH TWO WAVELENGHTS}

The use of multiple wavelengths in digital holography or interferometry is not new [15]. Applications in the field of Digital Holographic Microscopy [16, 17], vibration analysis [18], speckle reduction [19], and flow measurements have been demonstrated [20]. The redundancy of information produced by a multiple recording of the same scene enables enhancing the reliability of the experimental results. Here, the objective consists in separating a couple of holograms of a particle recorded at two different times into the two channels generated by each wavelength (i.e., $\lambda_{1}$ and $\lambda_{2}$ ). 


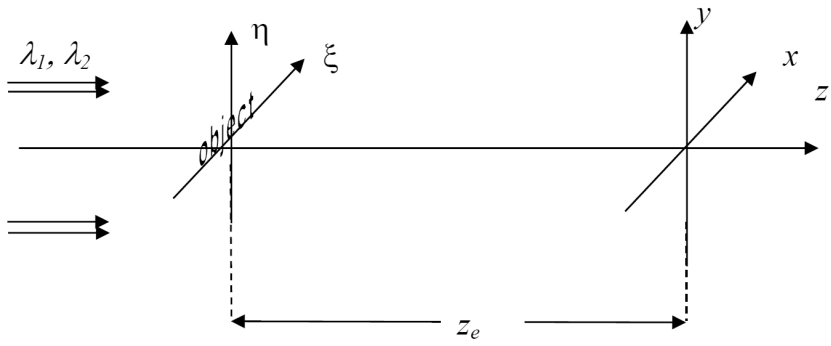

FIG. 1 Recording system of dual-wavelength digital in-line hologram $(\xi, \eta)$ : object plane; $(x, y)$ : sensor plane.

\subsection{Digital recording of the hologram}

In order to simplify the presentation of the basic idea, the convolution formalism is used for the description of the recording and reconstruction steps. Let $1-T(x, y)$ be the binary amplitude distribution of an opaque object illuminated by two monochromatic plane waves and located at a distance $z_{e}$ from a quadratic sensor, as shown by Figure 1 .

For a given wavelength $\lambda_{i}(\mathrm{i}=1$ or 2$)$, and from the HuygensFresnel integral, the complex amplitude in the detector plane can be expressed via the 2-D convolution operation [7]

$$
A_{z_{e}, \lambda_{i}}(x, y)=\left[(1-T) * * h_{z_{e}, \lambda_{i}}\right](x, y)
$$

where

$$
h_{z_{e}, \lambda_{i}}(x, y)=\frac{1}{i \lambda_{i} z_{e}} \exp \left[i \frac{\pi}{\lambda_{i} z_{e}}\left(x^{2}+y^{2}\right)\right]
$$

The intensity recorded by the sensor is deduced from the complex amplitude

$$
I_{z_{e}, \lambda_{i}}(x, y)=A_{z_{e}, \lambda_{i}}(x, y) \cdot \bar{A}_{z_{e}, \lambda_{i}}(x, y)
$$

Eq. (3), can be expressed as follows:

$$
I_{z_{e}, \lambda_{i}}(x, y)=1-\left[T * *\left(h_{z_{e}, \lambda_{i}}+\bar{h}_{z_{e}, \lambda_{i}}\right)\right](x, y)
$$

The term $\left|\left[T * * h_{z_{e}, \lambda_{i}}\right](x, y)\right|^{2}$ is omitted in Eq. (4) for simplification [13]. If we assume that both light sources $\left(\lambda_{1}\right.$ and $\lambda_{2}$, non mutually coherent) illuminate (at different time) the sample volume, the diffraction patterns accumulated by the CCD camera can be simply expressed as the sum of the intensity distributions:

$$
\begin{aligned}
I_{z_{e}, \lambda_{1}, \lambda_{2}}(x, y)=2- & {\left[T _ { 1 } * * \left(h_{z_{e}, \lambda_{1}}\right.\right.} \\
+ & \left.\left.\bar{h}_{z_{e}, \lambda_{1}}\right)+T_{2} * *\left(h_{z_{e}, \lambda_{2}}+\bar{h}_{z_{e}, \lambda_{2}}\right)\right](x, y)
\end{aligned}
$$

where

$$
\begin{aligned}
& T_{1}(x, y)=T\left(x-\Delta x_{0} / 2, y-\Delta y_{0} / 2\right) \\
& T_{2}(x, y)=T\left(x+\Delta x_{0} / 2, y+\Delta y_{0} / 2\right)
\end{aligned}
$$

Note that in Eq. (5), although a single particle is considered, two different object functions have been introduced because, in practice, the beams do not coincide exactly. This spatial shift has been intentionally introduced in order to illuminate the sample volume with two laser sources separated by a small angle $\alpha$. As a result, a given fixed object gives rise to a couple of holograms shifted in the $(x, y)$ plane by a small distance $\approx \alpha z_{e}$ (with $\alpha z_{e}=\sqrt{\Delta x_{0}^{2}+\Delta y_{0}^{2}}$ ). It is also interesting to note from Eq. (2) that $h_{z_{e}, \lambda_{2}}=h_{\frac{\lambda_{2}}{\lambda_{1}} z_{e}, \lambda_{1}}$. Consequently, Eq. (5) can be usefully rewritten as a double exposure hologram of the same object recorded under the wavelength $\lambda_{1}$ but located at the distances $z_{e}$ and $\frac{\lambda_{2}}{\lambda_{1}} z_{e}$ from the camera:

$$
\begin{aligned}
I_{z_{e}, \lambda_{1}, \lambda_{2}}(x, y)=2- & {\left[T_{1} * *\left(h_{z_{e}, \lambda_{1}}+\bar{h}_{z_{e}, \lambda_{1}}\right)\right.} \\
& \left.+T_{2} * *\left(h_{\frac{\lambda_{2}}{\lambda_{1}} z_{e}, \lambda_{1}}+\bar{h}_{\frac{\lambda_{2}}{\lambda_{1}} z_{e}, \lambda_{1}}\right)\right](x, y)
\end{aligned}
$$

Consequently, this configuration can be compared to the case of a double exposure hologram of a single particle that would have been shifted along the vector $\Delta_{0}=\left[\Delta x_{0}, \Delta y_{0},\left(\frac{\lambda_{2}}{\lambda_{1}}-1\right) z_{\mathcal{e}}\right]$ during the time interval between two pulses. It must also be observed that for sufficiently distinct wavelengths, the reconstructed images are spatially well separated in depth and each image can be processed independently. If a moving object is considered, the vector $\boldsymbol{\Delta}_{\mathbf{0}}$ has to be subtracted from the measured vector $\Delta$ to deduce the real 3D displacement.

\subsection{Hologram reconstruction}

Let us reconstruct the hologram images with the unique wavelength $\lambda_{1}$. The amplitude distribution in the reconstructed plane located at a distance $z_{r}$ from the camera is given by

$$
R_{z_{e}, \lambda_{1}}(x, y)=\left[I_{z_{e}, \lambda_{1}, \lambda_{2}} * * h_{z_{r}, \lambda_{1}}\right](x, y)
$$

Introducing Eq. (6) in Eq. (7) leads to

$$
\begin{aligned}
R_{z_{r}, \lambda_{1}}(x, y)=2- & {\left[T_{1} * *\left(h_{z_{e}, \lambda_{1}}+\bar{h}_{z_{e}, \lambda_{1}}\right)\right.} \\
& \left.+T_{2} * *\left(h_{\frac{\lambda_{2}}{\lambda_{1}} z_{e}, \lambda_{1}}+\bar{h}_{\frac{\lambda_{2}}{\lambda_{1}} z_{e}, \lambda_{1}}\right)\right] * * h_{z_{r}, \lambda_{1}}(x, y)
\end{aligned}
$$

Reconstructing the hologram consists in searching for those distances $z_{r}$ such that one of the convolutions $\overline{h_{z_{e}, \lambda_{1}}} * * h_{z_{r}, \lambda_{1}}(x, y)$ or $\overline{h_{\frac{\lambda_{2}}{\lambda_{1}} z_{e}, \lambda_{1}}} * * h_{z_{r}, \lambda_{1}}(x, y)$ is equal to the delta function $\delta(x, y)$.[8] Consequently, it can be easily seen that two images can be respectively reconstructed at the distances $z_{r 1}=z_{e}$ and $z_{r 2}=\frac{\lambda_{2}}{\lambda_{1}} z_{e}$. The intensity distribution in the reconstructed plane of the first image $\left(z_{r 1}=z_{e}\right)$ is

$$
\begin{aligned}
R_{z_{e}, \lambda_{1}}(x, y)=2 & -T_{1}(x, y)-\left[T_{1} * * h_{2 z_{e}, \lambda_{1}}\right](x, y) \\
& -\left[T_{2} * * \bar{h}\right. \\
z_{e}\left(\frac{\lambda_{2}}{\lambda_{1}}-1\right), \lambda_{1} & ](x, y) \\
& -\left[T_{2} * * h_{z_{e}\left(1+\frac{\lambda_{2}}{\lambda_{1}}\right), \lambda_{1}}\right](x, y)
\end{aligned}
$$

The usual reconstructed image $T_{1}$ and its conjugate are recovered. Note that unlike the off-axis configuration, the fringe pattern due to the defocused image of $T_{2}$ (i.e., $\left.\left[T_{2} * * \bar{h}_{z_{e}\left(\frac{\lambda_{2}}{\lambda_{1}}-1\right), \lambda_{1}}\right](x, y)\right)$ overlaps the image $T_{1}$. However, if we assume that the ratio $\frac{\lambda_{2}}{\lambda_{1}}$ is significantly different from unity, the fringes of this defocused image are spread over a large area and do not disturb the analysis of the image 1.

The second image (due to the source of wavelength $\lambda_{2}$ ) is reconstructed farther away, just behind the first image at $z_{r 2}=$ 


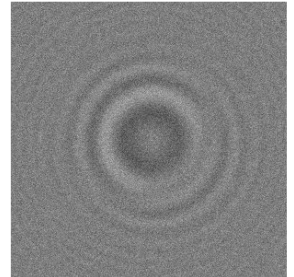

(a)

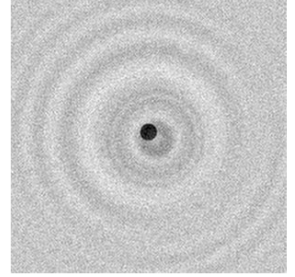

(b)

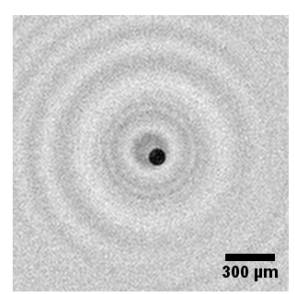

(c)
FIC. 2 Simulation of dual wavelength DIH. $z_{e}=100 \mathrm{~mm}, \mathrm{~d}=100 \mu \mathrm{m}, \alpha=4 \times 10^{-4}$, $\lambda_{1}=488 \mathrm{~nm}$ and $\lambda_{2}=640 \mathrm{~nm}$.Both images are reconstructed with the wavelength $\lambda_{1}$ (a) Hologram, (b) First image reconstructed at $z_{r 1}=100 \mathrm{~mm}$, (c) Second image reconstructed at $z_{r 2}=131 \mathrm{~mm}$. $\frac{\lambda_{2}}{\lambda_{1}} z_{e}$

$$
\begin{aligned}
R_{\frac{\lambda_{2}}{\lambda_{1}} z_{e}, \lambda_{1}}(x, y)= & -T_{2}(x, y)-\left[T_{2} * * h_{2 \frac{\lambda_{2}}{\lambda_{1}} z_{e}, \lambda_{1}}\right](x, y) \\
& -\left[T_{1} * * \bar{h}_{z_{e}\left(\frac{\lambda_{2}}{\lambda_{1}}-1\right), \lambda_{1}}\right](x, y) \\
& -\left[T_{1} * * h_{z_{e}\left(1+\frac{\lambda_{2}}{\lambda_{1}}\right), \lambda_{1}}\right](x, y)
\end{aligned}
$$

In addition, it is interesting to see that in the particular case of perfectly aligned beams and a fixed object, the object functions coincide $\left(T_{1}=T_{2}\right)$ and the real parts of the two last terms, representing the image due to the hologram recorded under the other wavelength (i.e., $-\left[T_{2} * * \bar{h} \bar{z}_{z_{e}\left(\frac{\lambda_{2}}{\lambda_{1}}-1\right), \lambda_{1}}\right](x, y)-\left[T_{2} * * h_{z_{e}\left(1+\frac{\lambda_{2}}{\lambda_{1}}\right), \lambda_{1}}\right](x, y)$ and $\left.-\left[T_{1} * * \bar{h}_{z_{e}\left(\frac{\lambda_{2}}{\lambda_{1}}-1\right), \lambda_{1}}\right](x, y)-\left[T_{1} * * h_{z_{e}\left(1+\frac{\lambda_{2}}{\lambda_{1}}\right), \lambda_{1}}\right](x, y) \quad\right)$ would be identical. Consequently, it can be concluded that apart from the twin images' fringes $\left[T_{1} * * h_{2 z_{e}, \lambda_{1}}\right](x, y)$ and $\left[T_{2} * * h_{2 \frac{\lambda_{2}}{\lambda_{1}} z_{e}, \lambda_{1}}\right](x, y)$ the reconstructed images are the same.

A similar reasoning could be carried out about a reconstruction with the other wavelength.

\subsection{Simulations and focusing method}

The following example illustrates the case of a $100 \mu \mathrm{m}$ fixed opaque disk recorded at $z_{e}=100 \mathrm{~mm}$ from a camera with a couple of beams with the wavelengths $\lambda_{1}=488 \mathrm{~nm}$ and $\lambda_{2}=640 \mathrm{~nm}$ and an angular separation $\alpha=4 \times 10^{-4} \mathrm{rad}$. From the hologram of Figure 2(a) and as expected from the developments given in Section 2.1, two images are reconstructed at the distances $z_{r_{1}}=100 \mathrm{~mm}$ and $z_{r_{2}}=131 \mathrm{~mm}$. As we can see in Figure 2(b) and 2(c), the images are mainly corrupted by the fringes due to the image recorded under the other wavelength and separated from the actual plane by a distance $z_{e}\left(\frac{\lambda_{2}}{\lambda_{1}}-1\right)$.

The search for the best focus plane is based on the method proposed in [21] for a Region Of Interest (ROI) around the reconstructed image of a particle. However, the variance of the imaginary part of a given reconstructed image at the distance $z_{r 1}$ (blue image) may be corrupted by the variance of the second image (supposed to be focused at $z_{r 2}$ ). In order to enhance the robustness of the focusing criterion, the variance of the real part of the reconstructed hologram has also been taken

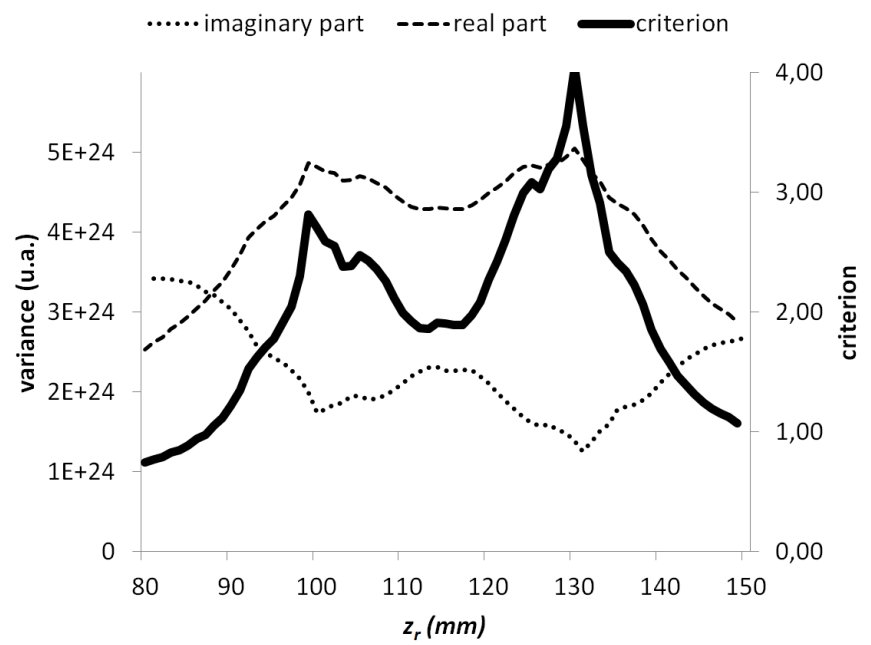

FIG. 3 Evolution of the focusing criterion on the hologram of Figure 2. The variances of the real and imaginary parts of the reconstructed image are calculated on a $60 \times 60$ ROI.

into account. The use of this complementary criterion is justified because when the best focus plane is reached, the image constrast is maximum inside the ROI. The best focus plane is considered as the distance $z$ that maximizes the ratio

$$
\text { criterion }=\frac{\operatorname{Var}\left\{\operatorname{Re}\left[R_{z_{r}, \lambda_{1}}(x, y)\right]\right\}_{R O I}}{\operatorname{Var}\left\{\operatorname{Im}\left[R_{z_{r}, \lambda_{1}}(x, y)\right]\right\}_{R O I}}
$$

The evolution of the variances of the real and imaginary parts of the reconstructed images are shown in Figure 3 for the example given in Figure 2.

As we can see in this example, this new criterion, which uses the whole complex amplitude of the reconstructed field, enables increasing the sharpness of the focusing peaks improving the reliability of the focusing method.

\section{EXPERIMENTAL RESULTS}

\subsection{Setup}

Two fiber coupled laser diodes (OXXIUS Laser, $\lambda_{1}=488 \mathrm{~nm}$ and Coherent CUBE $\lambda_{2}=640 \mathrm{~nm}$ ) are bundled in a device specially designed for this application and consisting in two monomode fibers juxtaposed in a single cladding (see Figure 4). The fiber cores are separated by a distance of $125 \mu \mathrm{m}$. The wavelength ratio $\left(\lambda_{2} / \lambda_{1}=1.311\right)$ is large enough so that the reconstructed images are well separated in depth. A second advantage of using two laser sources is that the time interval between each pulse can be easily reduced by up to $1 \mu \mathrm{s}$.

\subsection{Application to velocity measurements}

A feasibility experiment has been conducted with a particle sizing reticle consisting of more than 8000 opaque disks photographically etched on a glass substrate (see Figure 5(a)). The objects lie in the range $9-90 \mu \mathrm{m}$ and are randomly located within a circle of diameter $8 \mathrm{~mm}$. The standard reticle has been shifted $100 \mu \mathrm{m}$ along $\mathrm{x}$-axis between the two exposures (i.e. 


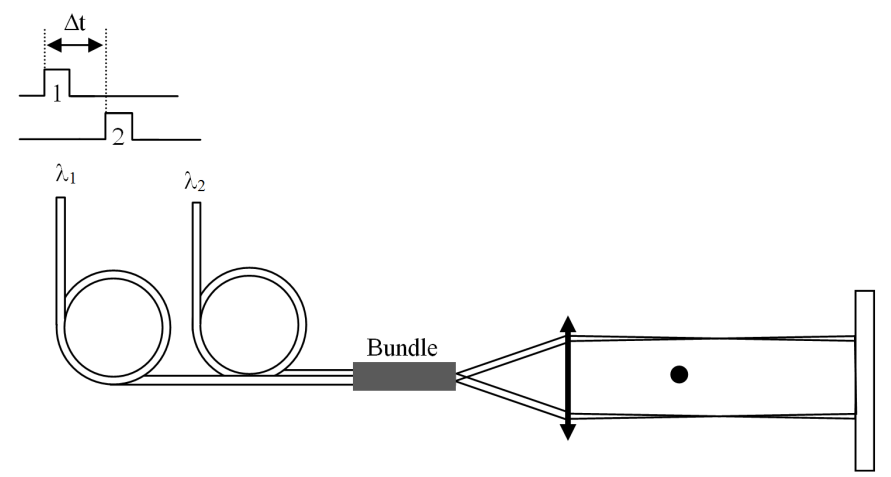

FIG. 4 Principle of a dual wavelength holographic recording setup for particle velocity measurements. In our experiment $\lambda_{1}=488 \mathrm{~nm}, \lambda_{2}=640 \mathrm{~nm}$.

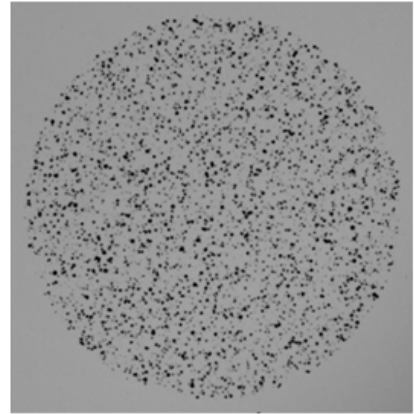

(a)

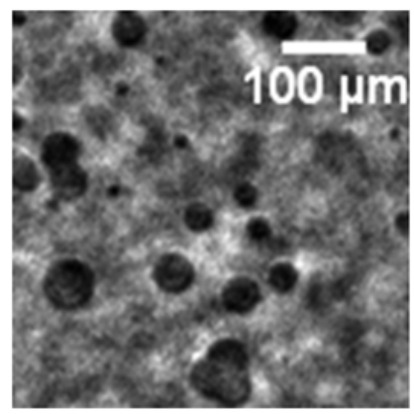

(c)

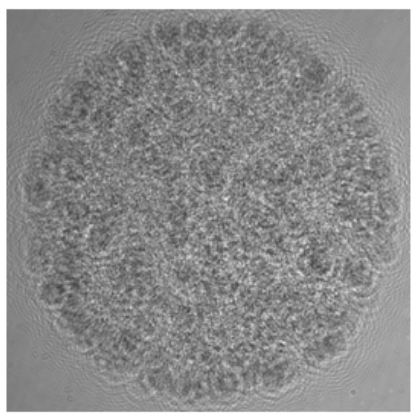

(b)

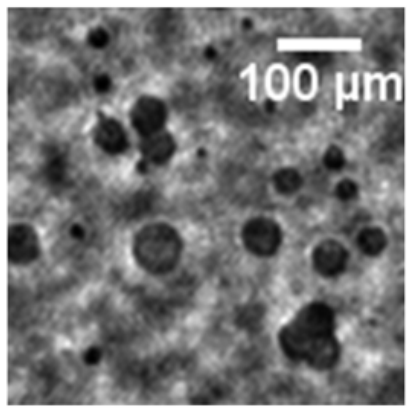

(d)
FIG. 5 Dual wavelength recording of a $(\Delta x=100 \mu \mathrm{m})$ moving particle field, (a) standard reticle used for the feasibility study, (b) 4096x4096 hologram: both patterns are recorded on the same frame, (c) $128 \times 128$ Rol cropped in the first image reconstructed at $z_{r 1}=285 \mathrm{~mm}$, (d) the same Rol cropped in the second image reconstructed at $z_{r 2}=373 \mathrm{~mm}$.

blue and red pulses). The hologram recorded with both wavelengths on a CCD camera (here 4096x4096 pixels are shown) is presented in Figure 5(b). It must be mentioned that in this feasibility experiment we have chosen to illuminate directly the CCD camera with divergent beams without introducing any lens in order to avoid chromatic aberrations. Note that in this particular optical configuration, the shift due to the separation of the two beams is not directly equal to $\alpha z_{e}$. Therefore a calibration of the experimental shift $\Delta_{0}$ was necessary. If a collimated beam is required, an achromatic optical system has to be used.

Figures 5(c) and (d) show both reconstructions $\left(z_{r 1}=285\right.$ $\mathrm{mm}$ and $z_{r 2}=373 \mathrm{~mm}$ ) within a small Region of Interest (ROI) cropped in the reconstructed volume (128x128 pixels). It can be observed that each image is reconstructed inde- pendently. Note that the experimental focusing distance ratio $\frac{z_{r 2}}{z_{r 1}}=1.309$ obtained by using our criterion is very close to the wavelength ratio (i.e. $\frac{\lambda_{2}}{\lambda_{1}}=1.311$ ). The lateral displacement $(\Delta x, \Delta y)$ was evaluated by using a standard crosscorrelation algorithm commonly used in PIV. The experimental values measured with this method $\left(\Delta x_{\text {meas }}=108.3 \mu \mathrm{m}\right)$ and $\left(\Delta y_{\text {meas }}=39.8 \mu \mathrm{m}\right)$ are in good agreement with the expected displacement (i.e. $\left(\Delta x_{t h}=111.2 \mu \mathrm{m}\right)$ and $\left(\Delta y_{t h}=42.7 \mu \mathrm{m}\right)$. Note that, as mentioned in subsection (2.1), this theoretical displacement $\left(\Delta x_{t h}, \Delta y_{t h}\right)$ is the vector sum of the reticle translation $(\Delta \mathrm{x}=100 \mu \mathrm{m}, \Delta \mathrm{y}=0)$ and the residual shift $\left(\Delta x_{0}, \Delta y_{0}\right)$ due to the beams' separation. The same process has been tested with a couple of holograms recorded with a moving reticle along the $z$-axis. Similar results obtained with $3 \mathrm{D}$ displacements confirm that this approach can be efficiently used for extracting 3D velocity vector fields without ambiguity in the sign of the velocity vector. Note that the feasibility demonstrated here with two wavelengths can be extended to three or more wavelengths.

\section{CONCLUSIONS}

A dual wavelength Digital Holographic system has been tested for an application to multiple exposure Digital In-line Holography. By separately recording the first and the second image of a particle field with two different wavelengths, we show that the reconstructed images can be easily processed in order to extract the 2D velocity vector fields. The experimental feasibility is demonstrated by using a standard reticle of particles illuminated by two fiber coupled laser diodes.

\section{ACKNOWLEDGEMENTS}

The authors are grateful for the financial support from program "3D" of the LABEX EMC3 (EnergyMaterials and Clean Combustion Center). This work was also supported by a grant from the "Region Haute-Normandie".

\section{References}

[1] H. Meng, G. Pan, Y. Pu, and S. H. Woodward, "Holographic particle image velocimetry: From film to digital recording," Meas. Sci. Technol. 15(4), 673 (2004).

[2] S. Coëtmellec, C. Buraga-Lefebvre, D. Lebrun, and C. Özkul, “Application of in-line digital holography to multiple plane velocimetry," Meas. Sci. Technol. 12(9), 1392 (2001).

[3] J. Sheng, E. Malkiel, and J. Katz, "Digital holographic microscope for measuring three-dimensional particle distributions and motions," Appl. Optics 45(16), 3893-3901 (2006).

[4] J. Soria, and C. Atkinson, "Towards 3C-3D digital holographic fluid velocity vector field measurement tomographic digital holographic PIV (Tomo-HPIV)," Meas. Sci. Technol. 19(7), 074002 (2008).

[5] F. Dubois, N. Callens, C. Yourassowsky, M. Hoyos, P. Kurowski, and 0 . Monnom, "Digital holographic microscopy with reduced spatial coherence for three-dimensional particle flow analysis," Appl. Optics 45(5), 864-871 (2006). 
[6] S. Coëtmellec, D. Lebrun, and C. Özkul, “Characterization of diffraction patterns directly from in-line holograms with the fractional Fourier Transform," Appl. Optics 41, 312-319 (2002).

[7] L. Onural, "Diffraction from a wavelet point of view," Opt. Lett. 18, 846-848,(1993).

[8] C. Buraga-Lefebvre, S. Coëtmellec, D. Lebrun, and C. Özkul, "Application of wavelet transform to hologram analysis: Threedimensional location of particles," Opt. Laser. Eng. 33(6), 409-421 (2000).

[9] F. Soulez, L. Denis, C. Fournier, E. Thiebaut, and C. Coepfert, "Inverse-problem approach for particle digital holography: Accurate location based on local optimization," J. Opt. Soc. Am. A 24(4), 1164-1171 (2007).

[10] W. Xu, M. H. Jericho, and H. J. Kreuzer, "Tracking particles in four dimensions with in-line holographic microscopy," Opt. Lett. 28, 164-166 (2003).

[11] N. Salah,, G. Godard, D. Lebrun, P. Paranthoën, D. Allano, and S. Coëtmellec, "Application of multiple exposure digital in line holography to particle tracking in a Benard von Karman vortex flow," Meas. Sci. Technol. 19(7), 074001 (2008).

[12] J. Lu, J. P. Fugal, H. Nordsiek, E. W. Saw, R. A. Shaw, and W. Yang, "Lagrangian particle tracking in three dimensions via single-camera in-line digital holography," New J. Phys. 10(12), 125013 (2008).

[13] D. Allano, M. Malek, F. Walle, F. Corbin, G. Godard, S. Coëtmellec, and D. Lebrun, "Three-dimensional velocity near-wall measurements by digital in-line holography: Calibration and results," Appl. Optics 52(1), A9-A17 (2013).
[14] R. J. Adrian, "Twenty years of particle image velocimetry," Exp. Fluids 39(2), 159-169 (2005)

[15] I. Yamaguchi, T. Matsumura, and J. I. Kato, " Phase-shifting color digital holography," Opt. Lett. 27(13), 1108-1110 (2002).

[16] J. Kühn, T. Colomb, F. Montfort, F. Charriere, Y. Emery, E. Cuche, P. Marquet, et al., "Real-time dual-wavelength digital holographic microscopy with a single hologram acquisition," Opt. Express 15(12), 7231-7242 (2007).

[17] J. Dohet-Eraly, C. Yourassowsky, and F. Dubois, "Refocusing based on amplitude analysis in color digital holographic microscopy," Opt. Lett. 39, 1109-1112 (2014).

[18] G. Pedrini, P. Fröning, H. J. Tiziani, and M. E. Gusev, "Pulsed digital holography for high-speed contouring that uses a two-wavelength method," Appl. Optics 38(16), 3460-3467 (1999).

[19] T. Nomura, M. Okamura, E. Nitanai, and T. Numata, "Image quality improvement of digital holography by superposition of reconstructed images obtained by multiple wavelengths," Appl. Optics 47(19), D38-D43 (2008).

[20] J. M. Desse, P. Picart, and P. Tankam, "Digital three-color holographic interferometry for flow analysis," Opt. Express 16(8), 5471$5480(2008)$.

[21] G. Pan, and H. Meng, "Digital holography of particle fields: Reconstruction by use of complex amplitude," Appl. Optics 42(5), 827-833 (2003). 